\title{
A new study of the supernova remnant G344.7-0.1 located in the vicinity of the unidentified TeV source HESS J1702-420
}

\author{
E. Giacani ${ }^{1,2}$, M. J. S. Smith ${ }^{3}$, G. Dubner ${ }^{1}$, and N. Loiseau ${ }^{4}$
}

\author{
${ }^{1}$ Instituto de Astronomía y Física del Espacio (CONICET-UBA), CC 67, Suc. 28, 1428 Buenos Aires, Argentina \\ e-mail: egiacani@iafe.uba.ar \\ 2 FADU, Universidad de Buenos Aires, Argentina \\ 3 XMM-Newton Science Operations Centre, ESAC/VEGA Space, Villafranca del Castillo, Spain \\ ${ }^{4}$ XMM-Newton Science Operations Centre, ESAC/INSA, Villafranca del Castillo, Spain
}

Received 22 February 2011 / Accepted 24 May 2011

\begin{abstract}
Context. The identification of counterparts of dark gamma-ray sources adds greatly to our understanding of their underlying astrophysical processes.

Aims. We investigate the physical properties of the supernova remnant (SNR) G344.7-0.1, to clarify whether it hosts a pulsar wind nebula (PWN), and the possible physical relationship between the SNR and the gamma-ray source HESS J1702-420.

Methods. The research was carried out based on new high-resolution radio images produced from archival ATCA and VLA data in combination with X-ray archival XMM-Newton data. The ambient interstellar medium was investigated in HI, ${ }^{12} \mathrm{CO}$ and mid IR $(\lambda 24 \mu \mathrm{m})$ with data from the public SGPS, CfA CO and MIPSGAL surveys, respectively.

Results. Based on the radio images and the comparison with X-ray and IR observations, we confirm that there is no PWN within G344.7-0.1; the observed emission highlights sites where the SN blast wave is encountering dense material. No radio counterpart is found for the X-ray object CXOU J170357.8-414302. The X-ray radiation completely fills in the interior of the SNR, being thermal in nature and originating in heated ejecta. From the spectral analysis it is inferred that G344.7-0.1 is the result of a core-collapse SN that exploded about $3000 \mathrm{yr}$ ago. On the basis of HI absorption and emission we redetermined its distance in $(6.3 \pm 0.1) \mathrm{kpc}$. From the study of the surrounding gas, we conclude that G344.7-0.1 has evolved within an HI bubble created by the SN precursor. This bubble is, in turn, part of a larger HI/IR ring created by the stellar wind of prior-generation stars, about $2.6 \times 10^{6} \mathrm{yr}$ ago. A second generation of stars formed in this compressed gas and about $3000 \mathrm{yr}$ ago one of these stars exploded, creating the SNR G344.7-0.1. This study suggests that G344.7-0.1 and its turbulent environment is a plausible counterpart for HESS J1702-420.
\end{abstract}

Key words. ISM: supernova remnants - ISM: individual objects: G344.7-0.1 - gamma rays: ISM $\mathrm{X}$-rays: individuals: HESS J1702-420

\section{Introduction}

Supernova remnants (SNRs) are a potential source of gammarays, either through inverse Compton scattering of electrons off ambient photons (Muraishi et al. 2000; Ellison et al. 2001) or through the decay of neutral pions created by the collision of energetic photons with dense ambient gas (Enomoto et al. 2002; Aharonian et al. 2006a). Additionally, several of the sources seen in very high energy gamma-rays are associated with pulsar wind nebulae (PWN), which result when the rotational energy of the neutron star, left after the SN event, is partially transformed into a highly relativistic wind of particles that is driven into the ambient medium, fuelling an extended non-thermal emission region. Together with shell type SNRs, PWNe are a dominant source population in the H.E.S.S. source catalog of VHE gamma-ray sources ${ }^{1}$. A complete understanding of the gamma-ray emission processes and their potential sources can only be achieved through a broadband study of the accelerators and their environs.

Supernova remnants, as such, exhibit a wide variety of properties and morphologies, which are the result of various causes,

\footnotetext{
1 http://www .mpi-hd.mpg.de/hfm/HESS/pages/home/ sources/
}

like the type of progenitor star, the explosion mechanism, the presence or not of a compact relic in its interior and, later in the evolution, also of the physical conditions of the surrounding interstellar medium (ISM). Multi-wavelength observations of SNRs and their environs are useful tools to understand the role of the different factors and their relevance in the emission distribution observed across the electromagnetic spectrum, including their connection with the high-energy emission.

G344.7-0.1 is a Galactic SNR classified on the basis of its radio morphology as a probable composite type (radio structure with a combination of shell and a centrally peaked component), with a size of about $8^{\prime}$ and a global spectral index $\alpha \sim-0.5$ $\left(S \propto v^{\alpha}\right)$ (Green 2009). The best radio maps available up to the present are those from the Very Large Array (VLA) obtained at $1.4 \mathrm{GHz}$ by Dubner et al. (1993) and at $843 \mathrm{MHz}$ by Whiteoak \& Green (1996), both with an angular resolution of about $40^{\prime \prime}$. These images revealed a circular shell structure, brighter towards the west, with an elongated maximum near the centre, suggested to be a nebula driven by the wind of an unseen pulsar (Dubner et al. 1993).

In the X-ray domain, the first detection of the SNR was reported by Yamauchi et al. (2005) based on ASCA observations, 
who found that the source consists of extended thermal emission, with maxima apparently correlated with the regions of bright radio emission. These authors fit the global spectrum to G344.7-0.1 with a thin thermal plasma model and an overabundance of S and Ca relative to the solar values. Recently, the thermal nature of the X-ray emission with enhanced abundances was confirmed through XMM-Newton observations (Giacani et al. 2010; Combi et al. 2010). Based on Chandra data, Combi et al. (2010) have also reported the existence of an unresolved soft X-ray source, CXOU J170357.8-414302, located near the geometrical centre of the radio remnant. This point source presents some characteristics of a central compact object (CCO), as observed in other SNRs. However, its association with a foreground K0 dwarf star seems to be more likely, based on the existence of infrared and optical counterparts, as well as on the absorbing column, which in the direction to this source is significantly different to the values estimated towards the SNR.

The SNR G344.7-0.1 is the only supernova remnant present in the adjacency of the very-high energy (VHE) source HESS J1702-420, a TeV source with a peculiar elongated shape discovered by Aharonian et al. (2006b). With increased observation time, Aharonian et al. (2008) confirmed that the source is detected at a significance level of $13 \sigma$ (Fig. 1) with a spectrum characterized by a power-law with index $2.1 \pm 0.1$. In spite of various studies carried out up to the present, it still belongs to the category of "dark" gamma-ray sources, without identified counterpart. The following have been suggested as possible counterparts: the pulsar PSR J1702-4128, lying at the northern edge of the gamma-ray emission, almost half a degree distant in the plane of the sky; the already mentioned SNR G344.7-0.1, located within the borders of the $\mathrm{TeV}$ source, about $20^{\prime}$ to the northeast of the VHE maximum; and three X-ray binaries in the vicinity of the VHE source, but outside the significant emission region. Among these possibilities, the pulsar PSR J1702-4128 was favoured by Aharonian et al. (2008) as the counterpart. However, in order for it to explain the observed gamma-ray emission several strong assumptions are required, including a conversion efficiency as high as $70 \%$ of the spin-down energy loss, an extremely asymmetric and quite large PWN (over $40 \mathrm{pc}$ at the assumed distance to PSR J1702-4128 of $5 \mathrm{kpc}$ ), and a rather high escape velocity for the pulsar of over $700 \mathrm{~km} \mathrm{~s}^{-1}$. Chang et al. (2008) carried out an X-ray study searching for a PWN associated with PSR J1702-4128, but the results are very marginal and inconclusive.

In this context, a new multi-wavelength study of the SNR G344.7-0.1 and its surrounding medium, is very opportune to shed some light on the nature of the SNR emission across the electromagnetic spectrum and its possible connection with HESS J1702-420. In this paper, we analyse unpublished radio data of the SNR G344.7-0.1 at $1.4 \mathrm{GHz}, 5 \mathrm{GHz}$, and $8.4 \mathrm{GHz}$ acquired with the Australia Telescope Compact Array (ATCA) and re-process the archival data at $1.4 \mathrm{GHz}$ from the VLA used in Dubner et al. (1993). We complement these radio observations by re-analysing the existing XMM-Newton data and, additionally, a study of the environs in the infrared at $24 \mu \mathrm{m}$ and in $\mathrm{HI}$ and ${ }^{12} \mathrm{CO}$ from public databases. Based on these data we produce new high-resolution radio images of the SNR and of its central nebula and compare this with the emission at other wavelengths, we analyse its radio and X-ray spectral properties, recalculate the distance to G344.7-0.1 and determine the characteristic parameters of the remnant based on the revised distance. We investigate the interstellar gas distribution in the surroundings of G344.7-0.1 and reveal that it evolves in a region with vestiges of past activity. Its relationship with the high-energy

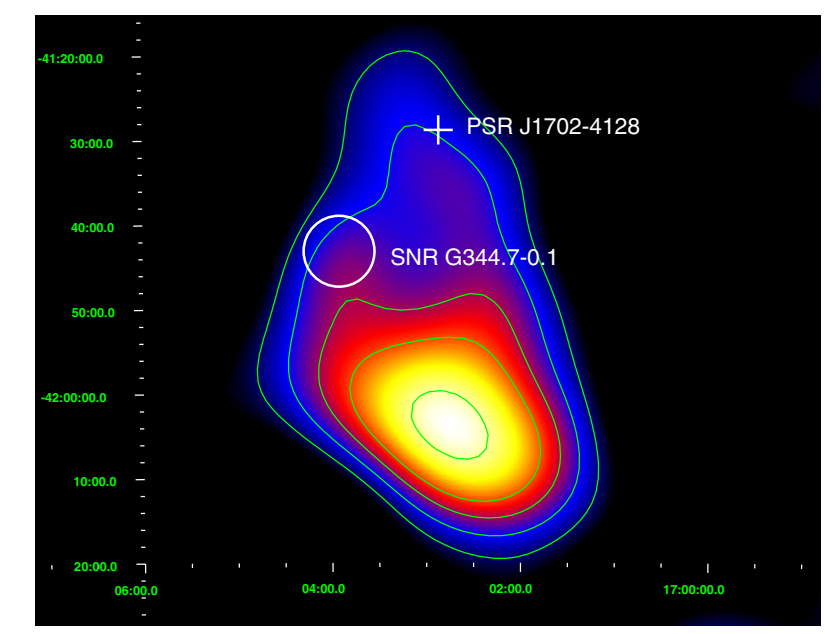

Fig. 1. Significance map of the very high energy gamma-ray source HESS J1702-420 (Aharonian et al. 2008) with the locations of the SNR G344.7-0.1 and PSR J1702-4128 indicated by a circle (with the same angular size as the SNR) and a plus sign, respectively. The outer contour corresponds to $13 \sigma$.

gamma-ray source HESS J1702-420 is investigated on the basis of the characteristics of the SNR and of the surrounding interstellar medium.

In Sect. 2 we describe the data used in this study. In Sect. 3 we present the results and discussion, and finally the conclusions are presented in Sect. 4.

\section{Data}

\subsection{New radio images of G344.7-0.1}

A radio image of G344.7-0.1 at $1.4 \mathrm{GHz}$ was produced using archival data corresponding to observations carried out with the VLA $^{2}$ in its hybrid DnC configuration on February 23, 1991 (project code AD620), and archival data from the Australia Telescope Compact Array (ATCA) acquired on April 4, 2004 in the 1.5A array and on May 6, 2004 with the interferometer operating in the $6 \mathrm{C}$ configuration. The interferometric data of both instruments were combined in the $u v$ plane. The resulting combined image has a synthesized beam of $8^{\prime \prime} \times 5^{\prime \prime}$. 6 , while the average $\mathrm{rms}$ noise is $0.3 \mathrm{mJy} /$ beam. It is worthwhile to note that this new image of G344.7-0.1 improves by a factor of four the angular resolution and by an order of magnitude the noise level with respect to any previously published image at this frequency.

To produce the image at $5 \mathrm{GHz}$ we used archival data from observations carried out with the ATCA telescope operating in the 750 D configuration on July 19, 1999. In this case, the longest baselines (i.e. all correlations with the sixth antenna) were excluded to enhance the surface brightness sensitivity. The final image has an angular resolution of $13^{\prime \prime} \times 10^{\prime \prime}$ and an rms noise of $0.2 \mathrm{mJy} /$ beam. This image is especially useful to investigate in detail the radio emission of the bright central region of the remnant. The surrounding radio shell (with a size of $\sim 8^{\prime}$ ) is barely detectable at this frequency because, in addition to its intrinsic faintness, the image suffers from attenuation produced near the primary beam edge ( $10^{\prime}$ at this frequency). The $8.4 \mathrm{GHz}$ image was produced from archival ATCA 750D configuration data observed in July 19 1999. In this case, the angular resolution is

\footnotetext{
2 The Very Large Array of the National Radio Astronomy Observatory is a facility of the National Science Foundation operated under cooperative agreement by Associated Universities, Inc.
} 
$10^{\prime \prime} \times 5^{\prime \prime}$ and the rms noise is $0.1 \mathrm{mJy} /$ beam. These latter observations are used to search for point-like sources. Data processing at all frequencies were carried out using MIRIAD software package.

\subsection{XMM-Newton data of G344.7-0.1}

We obtained X-ray images and spectra of G344.7-0.1 using public data of the XMM-Newton observatory. The data were processed with SAS version 10 and resulting spectra were analysed with Xspec version 11.3.2ag. We used EPIC data from the three separate occasions that the G344.7-0.1 field was observed by XMM-Newton: obsid 0111210101 on September 15, 2000 with the PN camera operating in Extended Full Frame Mode; obsid 0111210401 on August 28, 2001, with the PN in Extended Full Frame Mode and the MOS cameras in Full Frame Mode, and obsid 0506410101 September 13, 2007 with the three EPIC cameras in Full Frame Mode.

The total net exposure times after soft proton flare screening are $30.4 \mathrm{ks}$ and $38.9 \mathrm{ks}$ for the PN and MOS cameras respectively.

\subsection{The surrounding medium}

The neutral hydrogen around G344.7-0.1 was investigated using data from the Southern Galactic Plane Survey (SGPS) which provides HI data of the southern Milky Way obtained with the Australia Telescope Compact Array and Parkes Radiotelescopes (McClure-Griffiths et al. 2005). These data have an angular resolution of $2^{\prime}$, a velocity resolution of $0.82 \mathrm{~km} \mathrm{~s}^{-1}$ and an rms sensitivity of $1 \mathrm{~K}$.

The mid-infrared emission used to investigate the interaction of the SNR shock with the surrounding medium was extracted from the Multiband Imaging Photometer System (MIPS) (Rieke et al. 2004) aboard the Spitzer Space Telescope survey at $24 \mu \mathrm{m}$ (MIPSGAL). The images has an angular resolution of 6", a sampling of 1". 25 and a point source sensitivity of $1.7 \mathrm{mJy}$.

We also explored the ${ }^{12} \mathrm{CO}$ emission in the region using the CO Galactic Plane Survey (Dame et al. 2001) for which the angular resolution and spectral resolution are 8.8 and $1.3 \mathrm{~km} \mathrm{~s}^{-1}$, respectively. Although in this case the angular resolution is insufficient to carry out a detailed analysis of the molecular gas associated with the SNR, it is useful as complementary data to investigate the ISM properties in connection with the VHE source.

\section{Results and discussions}

\subsection{The morphology of SNR G344.7-0.1}

Figure 2 shows a comparison of the SNR G344.7-0.1 emission distribution in different wavelength. The images display the same field of view with identical spatial scale, with the exception of the upper-right image, which shows an enlargement of the central part of the SNR as detected at $5 \mathrm{GHz}$ (the area delimited by the green square in the image at $1.4 \mathrm{GHz}$ ). The green contours serve to highlight the brightest $1.4 \mathrm{GHz}$ emission; they are overlayed on each of the images to facilitate the inter-band comparison.

The dominant aspect of the overall morphology of G344.7-0.1 in the radio domain is the strong contrast between the bright western and central part with the much fainter eastern half of the SNR, as was already noted in the previously published images. The high fidelity of the new $1.4 \mathrm{GHz}$ image reveals that the shell, with a diameter of about $8^{\prime}$, is almost complete and quite perfectly spherical. Towards the SE border the radio emission vanishes. The bright radio synchrotron emission towards the centre of the remnant appears to be quite structured at both observed radio frequencies. This feature has a bright clump centred at $17^{\mathrm{h}} 03^{\mathrm{m}} 55^{\mathrm{s}} .8,-41^{\circ} 40^{\prime} 44^{\prime \prime} .6$ and several arcs with different orientations. Weaker diffuse emission with a mean intensity of $0.8 \mathrm{mJy} / \mathrm{beam}$ fills in the western half of the remnant. The new, sensitive radio image does not show any trace of a radio counterpart for the soft X-ray compact object CXOU J170357.8414302 discovered by Combi et al. (2010), down to a level of $0.3 \mathrm{mJy} /$ beam. A careful inspection of the $5 \mathrm{GHz}$ and $8 \mathrm{GHz}$ images also reported negative results.

To produce the X-ray image of G344.7-0.1 included in Fig. 2 (lower-left panel), the MOS and PN images were merged and smoothed with a 5" (FWHM) Gaussian kernel. The X-ray emission appears spherically distributed with two maxima, the brightest of which is at the centre of the SNR and a secondary, with an arched shape, towards the NW. The radio contours overlapping the $\mathrm{X}$-ray emission reveal that the maxima in radio and in thermal X-rays are uncorrelated, since the central radio nebula fills in the gap between the two X-ray brightest regions.

Finally, at $\lambda 24 \mu \mathrm{m}, \mathrm{G} 344.7-0.1$ shows a filamentary structure. In this case the IR maxima are very well correlated with the radio synchrotron peaks.

Figure 3 shows in a false-colour scheme the comparison of the multispectral emission of the SNR G344.7-0.1. The upperleft image displays the radio $1.4 \mathrm{GHz}$ (cyan)-X-ray (red) comparison. Purple and white show the regions where both emission distributions overlap. As previously noticed by Yamauchi et al. (2005) and Combi et al. (2010) X-ray emission is completely interior to the radio shell, filling it in its totality. However, in contrast with these previous works that concluded that X-ray emission correlates very well with regions of bright radio and IR emission, our new high fidelity image shows, as mentioned above, that the brightest features in radio are, in general, spatially uncorrelated with the most intense X-ray emission.

Figure 3 upper-right shows the comparison of radio (in red) and mid- IR $24 \mu \mathrm{m}$ emission (in green). Infrared emission reveals the presence of shock-heated swept up dust from the circumstellar or interstellar medium, and potentially dust created by the supernova itself. Striking IR filaments are observed ahead of the radio shock position in the southwest and northeast limbs, revealing a dust envelope around the SNR which is being heated by the shock wave. Similar features are observed for example in Tycho and Kepler SNRs, where IR-24 $\mu$ m emission traces collisionaly heated dust, where grains are heated by collisions with ions and electrons. It is important to note that the bright central elongated radio maximum is also bright in $24 \mu \mathrm{m}$ (shown in yellow in Fig. 3 upper-right), highlighting a site where it is very probable that the $\mathrm{SN}$ shock is interacting with clumpy, dusty circumstellar and/or interstellar gas located in front of the expanding bubble.

Figure 3 lower-left shows the comparison between the X-ray emission (in blue) and the IR emission (in green). The emission in both ranges cover approximately the same volume inside the SNR, except for the bright outer filaments observed in IR which are not observed in X-ray. Besides, as mentioned above, the maxima are not coincident.

Finally, Fig. 3 lower-right displays the combination of radio, IR and X-ray emissions, confirming the general picture of G344.7-0.1 as a synchrotron shell detected in radio wavelengths, filled with hot gas that emits thermal X-rays, and warm dust, collisionally heated by the expanding SN shock emitting in mid-IR. The central maxima observed in radio and infrared 


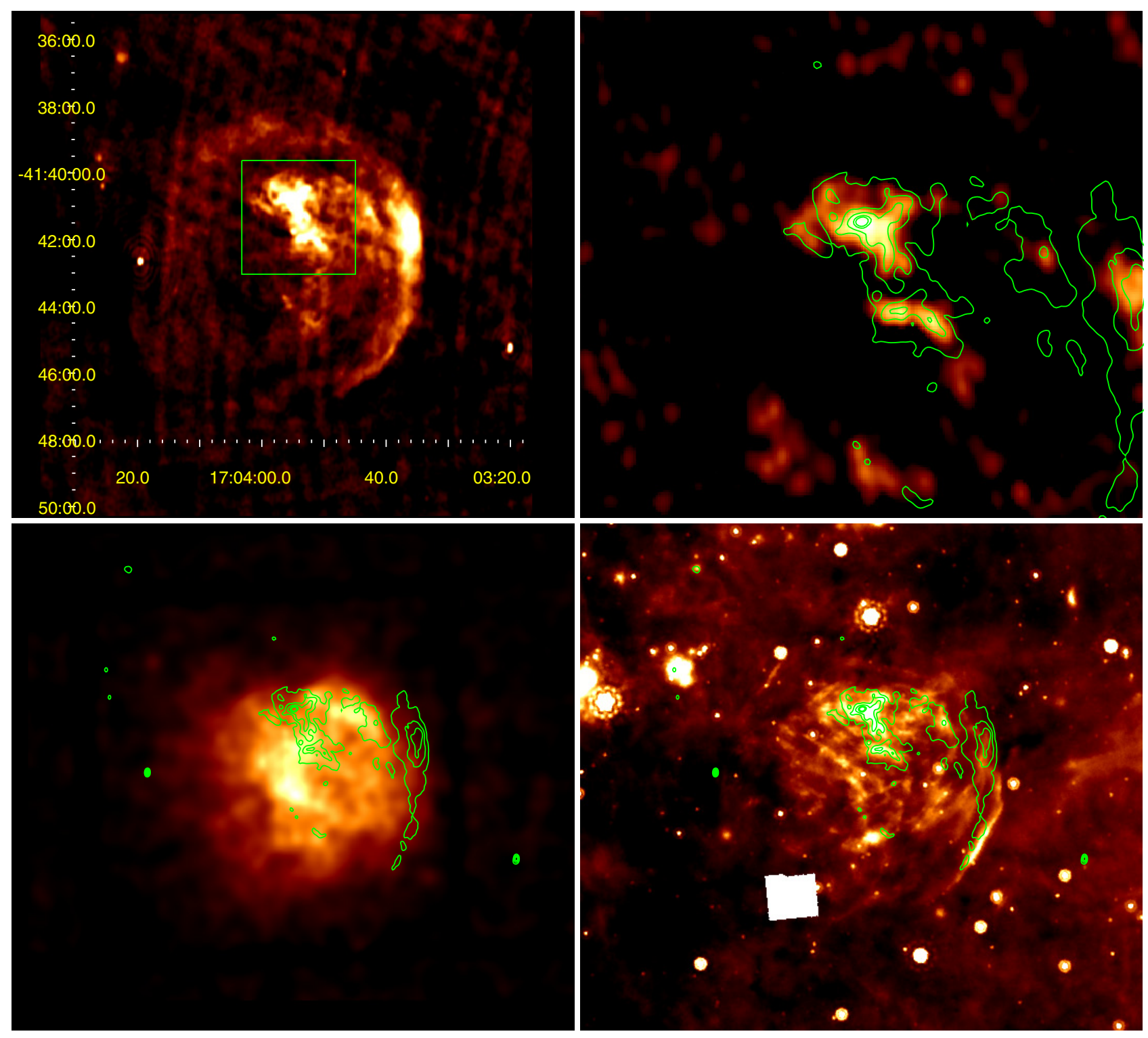

Fig. 2. Upper-left: ATCA and VLA radio image of G344.7-0.1 at 1.4 GHz. The green square shows the area enlarged in the contiguous panel; upper-right: the central nebula as observed with ATCA at $5 \mathrm{GHz}$; lower-left: XMM-Newton EPIC image of G344.7-0.1 in the 0.3-8.0 keV energy band; lower-right: mid-infrared $24 \mu \mathrm{m}$ image of G344.7-0.1 from Spitzer MIPSGAL; the blank square has data flagged as problematic.

is probably illuminating the location of a shocked dense cloud, which would be absorbing the X-ray emission.

\subsection{Spectral studies}

\subsubsection{Radio spectral index of SNR G344.7-0.1}

In order to investigate the nature of the radio emission seen near the centre of G344.7-0.1 and clearly discriminate if it is the synchrotron nebula driven by an unseen pulsar or a consequence of the interaction of the expanding SNR shock with a foreground cloud, we calculated its radio spectral index between 1.4 and $5 \mathrm{GHz}$.

To carry out this study we first of all matched both images in all aspects (e.g., $u v$ coverage, centre and pixel alignment), and degraded the spectral map to a spatial resolution of $15^{\prime \prime} \times 15^{\prime \prime}$ to obviate spurious small scale effects. By clipping both images at a $3 \sigma \mathrm{rms}$ noise level to avoid spectral index determination in regions with very faint radio emission, and by integrating the same area in both images to estimate the flux density, we obtained a mean radio spectral index $\alpha \sim-0.3 \pm 0.1$ between 1.4 and $5 \mathrm{GHz}$, where appropriate corrections were applied to take into account unrelated background contribution. To confirm these results, we also computed the spectral index using the T-T plot technique (Costain 1960; Turtle et al. 1962), which has the advantage that it is not sensitive to a difference in the zero levels between the maps at the two frequencies. A linear fit to the plot gives a measure of the spectral index. For correlation very close to one, the slope of the linear fit is independent of which of two frequencies is used as abscissa. But in general, this is not true, therefore it is necessary to compute the two different slopes obtained when each of the two frequencies is used as abscissa. The spectral indices using the average of both slopes are $\alpha=-0.2 \pm 0.1$ for the central radio emission, where the error encloses the values obtained for the different slopes.

For a PWN a flat radio spectrum with $\alpha$ between $\sim-0.3$ and 0 is expected (Gaensler \& Slane 2006). The spectrum obtained is in principle compatible with the PWN predicted values. A key confirmation of the PWN scenario would be the detection of a pulsar, either in radio or in X-rays. In the Parkes 

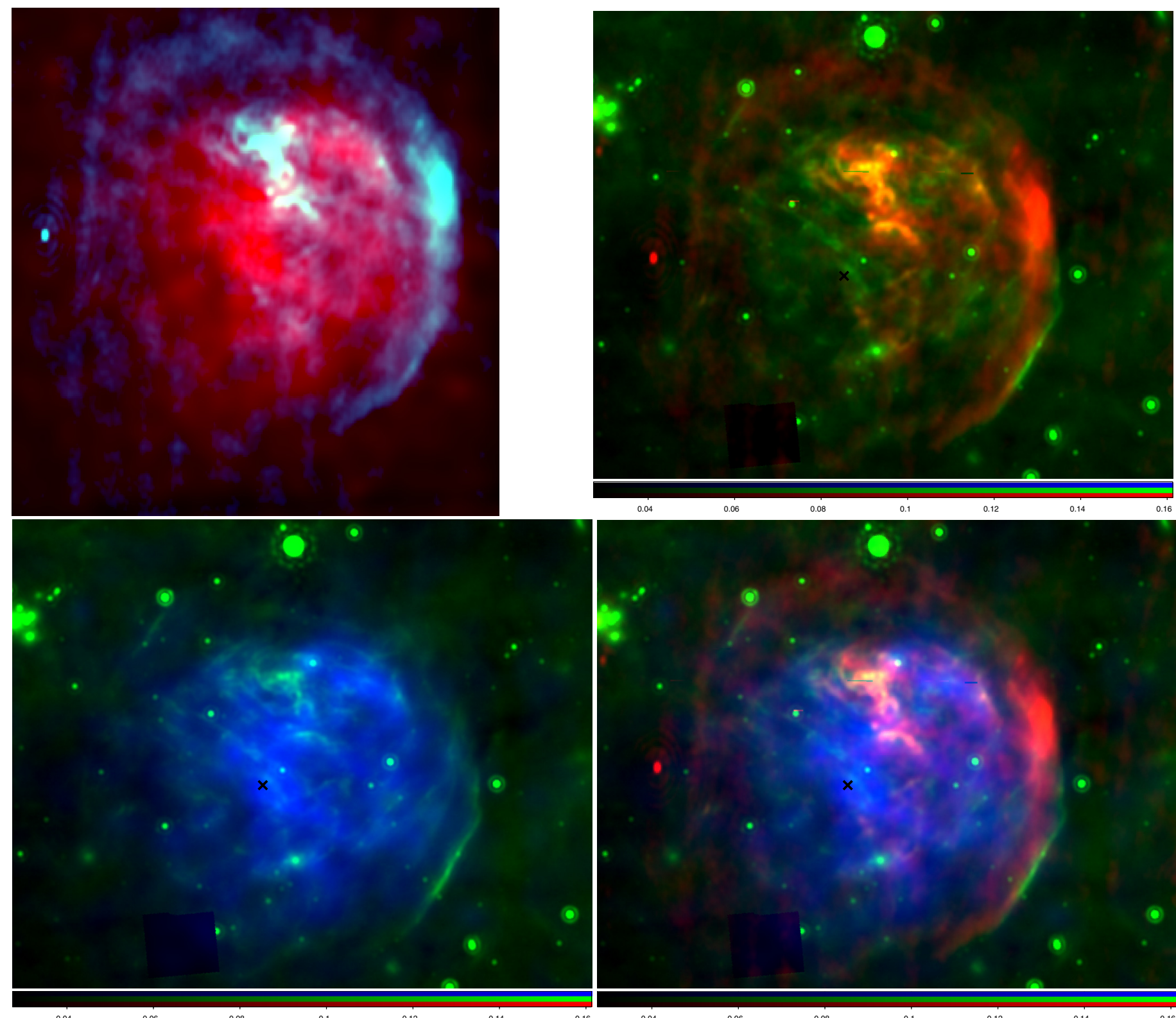

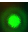
0.16

0.04

0.06

0.12

0.04

0.08

0.1

0.12

0.14

Fig. 3. Two-color combinations of the emission associated with G344.7-0.1 in the different spectral ranges. Upper left: radio (red) with X-ray (cyan); upper right: radio (red) and IR-24 $\mu \mathrm{m}$ (green); lower left: IR-24 $\mu \mathrm{m}$ (green) and X-ray (blue); lower right: three-bands combination, radio (red), IR-24 $\mu \mathrm{m}$ (green) and X-ray (blue). The small black cross indicates the position of the X-ray source CXOU J170357.8-414302.

multi-beam survey no pulsar close to G344.7-0.1 has been found. We detected a non-pulsating point-like radio source located at $\left(17^{\mathrm{h}} 03^{\mathrm{m}} 54.9,-41^{\circ} 41^{\prime} 44^{\prime \prime} \cdot 6\right)$, about 1.5 northwest to the X-ray point source CXOU J170357.8-414302. This radio source has a flux density of $2 \mathrm{mJy}$ at $1.4 \mathrm{GHz}$ and it has not been detected at $5 \mathrm{GHz}$ down to a noise level of $0.2 \mathrm{mJy} / \mathrm{beam}$, thus setting an upper limit for its radio spectral index to about -1.8 , which is not very different from the mean radio pulsars spectral indices (Taylor et al. 1993). Therefore, though the PWN hypothesis is very unlikely, it cannot be completely discarded without further studies searching for pulsations and polarization.

Another possibility to consider is that the presence of optically thick thermal gas placed along the line of sight to the SNR may absorb a steeper synchrotron background emission (the SNR emission with $\alpha \sim-0.5$ ), thereby flattening the apparent spectral index. The presence of such thermal absorption usually manifests itself as a turnover of the radio spectrum at low frequencies (less than $\sim 100 \mathrm{MHz}$ ). As we are observing the effect at a frequency of $1.4 \mathrm{GHz}$, such absorption would require the presence of very thick material in front of the SNR, which seems unlikely in view of the lack of dark clouds as can be seen in optical images of the region. A more appropriate explanation for the spectral flattening that we observe towards this central nebula is that the expanding SN shock wave has run into a wall of molecular gas and loses energy radiatively, appearing as optically thin thermal emission (with a thermal spectral index of say $\sim-0.1 \sim-0.2$ ) blended with the background nonthermal emission of the SNR. A similar situation is observed in the SNR IC 443, where Castelletti et al. (2011, in press) have shown that a fast dissociating J-type shock across an interacting molecular cloud reflects in a flat radio spectrum. The hypothesis of a shocked cloud in front of G344.7-0.1 is further supported by the picture derived from the multiwavelength images, where radio and IR maxima would be marking the location of the cloud 
which, in turn, is observed as an absorption zone in X-rays. The proposed cloud might well be part of a pre-existing stellar wind bubble blown up by the massive precursor star (see Sect. 3.3).

In summary, from this study we conclude that the more plausible explanation for the central feature in G344.7-0.1 is that it is the result of the interaction of the expanding SN blast wave with inhomogeneous ambient medium, dropping the hypothesis of a putative PWN created by an unseen pulsar.

\subsubsection{X-ray spectroscopy of the SNR G344.7-0.1}

We extracted the MOS1, MOS2, and PN spectra of both observations from a circular region of radius 3.6 centred on $17^{\mathrm{h}} 03^{\mathrm{m}} 51^{\mathrm{s}} .8,-41^{\circ} 42^{\prime} 40^{\prime} \cdot 0$, which covers the entire observed $\mathrm{X}$-ray emission. The background spectra of the three instruments were taken from source free regions of the fields. Due to the extended nature of the source, and its location in different areas of the detector from observation to observation, the PN source events were further corrected using suitably scaled detector backgrounds obtained from observations performed with the Closed filter configuration. The resulting global SNR spectrum contains a total of $6 \times 10^{4}$ net source counts.

The data were simultaneously fit in the $0.9-7 \mathrm{keV}$ band with a non-equilibrium ionization collisional plasma model, assuming a constant temperature and single ionization parameter (VNEI, Borkowski et al. 2001), combined with an absorption component. With the associated NEIVERS parameter set to 2, the model uses the ionisation fractions of Mazzotta et al. (1998) and APED (Smith et al. 2001) to generate the resulting spectrum. All fit parameters except the normalisation were tied between MOS and PN, and between the various observations. The normalisation was left to vary independently so as to allow for the differing coverage of the remnant with respect to chip gaps and bad pixels from observation to observation.

In the model fitting, initially the elemental abundances were fixed to the solar values of Anders \& Grevesse (1989). Successive freeing of the abundance parameters of $\mathrm{Mg}, \mathrm{Si}, \mathrm{S}$, $\mathrm{Ar}, \mathrm{Ca}$ and $\mathrm{Fe}$ led to statistically significant improvements of the model fit (>99.99\% likelihood per freed parameter on the basis of an F-test). Other elemental abundances are not significantly different from their solar values.

An additional zero-width Gaussian component was needed to model the $\mathrm{Fe} \mathrm{K}$ emission, resulting in a line centroid at $6.44 \pm$ $0.05 \mathrm{keV}$, which is consistent with emission caused by $\mathrm{Fe} \mathrm{K} \alpha$ fluorescence. We obtained a statistically good fit with a reduced $\chi^{2}$ of 0.98 for 2244 degrees of freedom, the results of which are listed in Table 1 and are plotted in Fig. 4.

As can be seen, the best fit requires significant over abundances of $\mathrm{S}, \mathrm{Si}, \mathrm{Ar}$, and $\mathrm{Ca}$ with respect to their solar values, which indicates the presence of ejecta material.

We also compared our fit with that reported by Combi et al. (2010) using the PSHOCK model (Borkowski et al. 2001). The PSHOCK model fit is quite good overall, although its reduced $\chi^{2}$ of 1.15 (for 2251 degrees of freedom) is worse than that obtained with the VNEI model. In particular, the PSHOCK model shows substantial systematic residuals around $3.1 \mathrm{keV}$ (corresponding to the atomic transition energy of Ar XVII), where the model significantly underestimates the data. This is probably due to the fact that, in the fit, the PSHOCK abundance parameter is driven by the $\mathrm{Mg}, \mathrm{Si}$, and $\mathrm{S}$ elemental abundances, and therefore does not fully account for the higher abundance of Ar.

We did not find obvious spectral variation across the remnant, probably due to the limited photon statistics.
Table 1. The best-fit model of the global X-ray spectrum of G344.7-0.1.

\begin{tabular}{lc}
\hline \hline Parameters & Values \\
\hline$k T\left(\mathrm{keV}^{-0}\right)$ & $1.46_{-0.07}^{+0.10}$ \\
$\tau\left(\mathrm{s} \mathrm{cm}^{-3}\right)$ & $7.8 \pm 1.0 \times 10^{10}$ \\
$\mathrm{Mg}$ abundance & $0.26 \pm 0.1$ \\
Si abundance & $1.25 \pm 0.1$ \\
$\mathrm{~S}$ abundance & $1.94 \pm 0.1$ \\
Ar abundance & $2.69 \pm 0.3$ \\
$\mathrm{Ca}$ abundance & $4.7_{-1}^{+2}$ \\
Fe abundance & $0.0_{-0.0}^{+0.2}$ \\
$N_{\mathrm{H}}\left(\mathrm{cm}^{-2}\right)$ & $4.5 \pm 0.2 \times 10^{22}$ \\
Normalisation & $0.042 \pm 0.005$ \\
\hline
\end{tabular}

Notes. The elemental abundances listed are those considered to be free parameters of the model. Other abundances are fixed to their solar values. The normalisation is averaged over the best fit values of each instrument and observation, and is defined as $10^{-14} / 4 \pi D^{2} \times \int n_{\mathrm{H}} n_{\mathrm{e}} \mathrm{d} V$, where $D$ is the distance to the source in units of $\mathrm{cm}$, and $n_{\mathrm{e}}$ and $n_{\mathrm{H}}$ are the electron and hydrogen densities respectively, in units of $\mathrm{cm}^{-3}$.

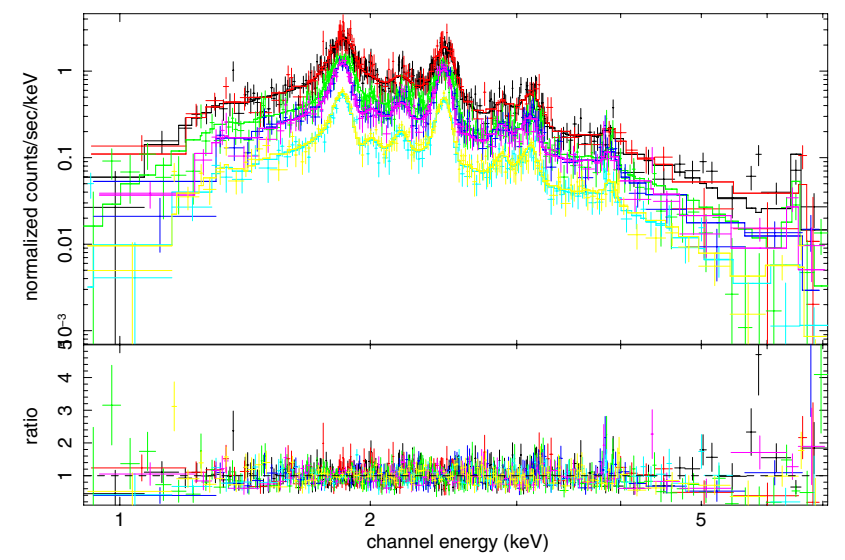

Fig. 4. EPIC spectra and data-to-model ratios of G344.7-0.1 for observations 0111201010 (PN black), 0111210401 (PN red, MOS1 blue, MOS2 magenta), and 0506410101 (PN green, MOS1 cyan, MOS2 yellow).

Based on the emission measure $(\mathrm{EM})$ of $1.99 \times 10^{58} \mathrm{~cm}^{-3}$ determined from our spectral fitting, we can calculate the electron density of the plasma to be $n_{\mathrm{e}}=(\mathrm{EM} / V)^{1 / 2} \simeq 0.8 \mathrm{~cm}^{-3}$, where $V$ is the volume of the X-ray emitting plasma considered in this case to be a sphere of 3.6 of radius, and the hydrogen number density, $n_{\mathrm{e}}=n_{\mathrm{H}}$, for simplicity. In addition we can estimate the lower limit of the mass of the X-ray emitting gas in $\simeq 22 M_{\odot}$, assuming cosmic abundances as a first approximation. For a shock temperature of $k T=1.46 \mathrm{keV}$, the shock velocity would be $v_{\mathrm{s}}=\left(16 k T / 3 \mu \mathrm{m}_{\mathrm{H}}\right)^{1 / 2} \simeq 1100 \mathrm{~km} \mathrm{~s}^{-1}$ (with a mean atomic weight $\mu=0.61$ ). For the preceding numerical values, we estimated the supernova explosion energy $E$ to be about $2.6 \times 10^{50} \mathrm{erg}$. This value of the explosion energy is lower than the canonical value of $10^{51} \mathrm{erg}$, but similar to that found in other SNRs from their X-ray emission, e.g. G272.2-3.2 (Harrus et al. 2001), and G299.2-2.9 (Park et al. 2007). Probably, as was suggested recently by Castro et al. (2011), some of the energy from the shock was lost to cosmic ray acceleration during the earlier evolutionary state of the remnant. The acceleration process shut off when the SNR entered into the Sedov-Taylor phase and consequently we infer a much lower explosion energy based on the current observed properties of the SNR shock. Based on the NEI fit to the spectrum of the entire remnant in the 
0.9-7.0 keV energy band, we estimate the X-ray luminosity to be $L_{\mathrm{X}} \sim 2.25 \times 10^{35} \mathrm{erg} \mathrm{s}^{-1}$ for a distance of $6.3 \mathrm{kpc}$.

The age of the remnant, estimated to be $\tau / n_{\mathrm{e}}$, is found to be $3000 \mathrm{yr}$. This age is in excellent agreement with the age estimated by applying Chevalier (1974)'s model for a SNR in Sedov phase, $t=\left(R_{\mathrm{pc}} / 21.9\right)^{3.23} \times 10^{5} \mathrm{yr} \simeq 2900 \mathrm{yr}($ where $R \simeq 7.3 \mathrm{pc}$ is the radius of the radio remnant at an assumed distance of $6.3 \mathrm{kpc}$, see Sect. 3.3).

In summary, from our X-ray study we can conclude that G344.7-0.1 is a relatively young SNR with thermally dominated X-ray emission and enhanced metal abundances indicative of ejecta origin.

\subsection{A new determination of the distance and the environs of G344.7-0.1}

The distance is a crucial variable to properly estimate the characteristic parameters of G344.7-0.1 and therefore analyze its nature and the possible connection with the H.E.S.S. source. The only previous estimate of distance for this SNR was done by Dubner et al. (1993), who calculated a distance of about $14 \mathrm{kpc}$ to G344.7-0.1 on the basis of the $\Sigma-\mathrm{D}$ relationship; however this method is known to have large intrinsic dispersion and it is not reliable. Here we attempt to constrain the distance to G344.7-0.1 through absorption technique using the HI profiles extracted from the Southern Galactic Plane Survey (SGPS).

$\mathrm{HI}$ absorption profiles were traced in the direction to bright emission radio continuum regions in G344.7-0.1, and towards nearby regions devoid of continuum emission. We find that the most distant HI absorption feature towards G344.7-0.1 appears close to the radial velocity $v_{\mathrm{LSR}}=-120 \pm 1 \mathrm{~km} \mathrm{~s}^{-1}$. Using a Galactic circular rotation law with a flat rotation curve with solar constants $R_{0}=8.33 \mathrm{kpc}$ and $\Omega_{0}=27.2 \mathrm{~km} \mathrm{~s}^{-1} \mathrm{kpc}^{-1}$, this velocity referred to the local standard of rest (LSR) corresponds to the near and far kinematical distances of $\sim 6.3 \pm 0.1 \mathrm{kpc}$ and $\sim 9.7 \pm 0.1 \mathrm{kpc}$. We can therefore assume that $\sim 6.3 \mathrm{kpc}$ is the lower limit for the distance to G344.7-0.1. The upper limit to the distance can be determined from the Galactic tangent point velocity $v_{\text {LSR }} \sim-164 \pm 0.1 \mathrm{~km} \mathrm{~s}^{-1}$, which corresponds to a kinematic distance of $\sim 8 \mathrm{kpc}$. This sets the lower and upper limits to the distance of G344.7-0.1 as $\sim 6.3$ and $\sim 8 \mathrm{kpc}$, respectively.

An inspection of the HI distribution at velocities near $-120 \mathrm{~km} \mathrm{~s}^{-1}$ revealed the striking presence of an open HI shell that embraces most of the periphery of G344.7-0.1, especially around the northern and western sides of the SNR where the SNR's limb is brighter, and opens to the southeast, where the SNR vanishes breaking out into lower density interstellar gas. This HI shell can be detected approximately in the velocity range between -119 and $-110 \mathrm{~km} \mathrm{~s}^{-1}$ (see Fig. 5) which, taking into account the typical uncertainties of $\pm 7 \mathrm{~km} \mathrm{~s}^{-1}$ representative of the random motions of $\mathrm{HI}$ clouds in the Galaxy, is in good agreement with the velocity derived on the basis of absorption profiles. Therefore, it is very likely that the HI gas in this velocity range is not only spatially, but also physically associated with G344.7-0.1. By adopting $v_{\mathrm{LSR}} \sim-115 \mathrm{~km} \mathrm{~s}^{-1}$ as the systemic velocity for the $\mathrm{HI}$ shell, it translates into a kinematical distance of $6.3 \pm 0.1 \mathrm{kpc}$, resolving the ambiguity in favour of the closer distance. Thus, based on the HI absorption and on the finding of an associated HI emission feature, we determined a more precise distance to G344.7-0.1 as $6.3 \pm 0.1 \mathrm{kpc}$.

A further constraint on the distance can be obtained by a comparison between the absorbing material across the remnant estimated from ${ }^{12} \mathrm{CO}$ and $\mathrm{HI}$ data and the foreground absorption derived from our X-ray data. The atomic and molecular gas

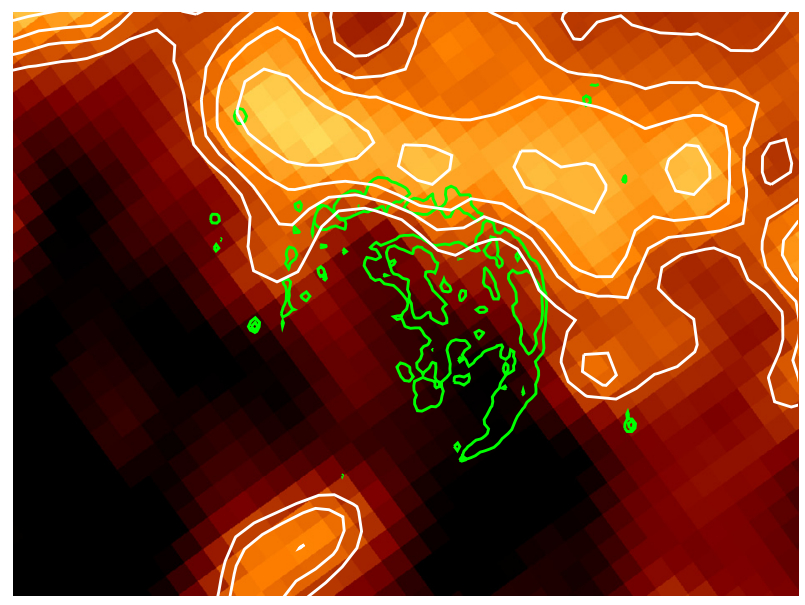

Fig. 5. The neutral hydrogen distribution around G344.7-0.1 near $v_{\mathrm{LSR}} \sim-115 \mathrm{~km} \mathrm{~s}^{-1}$ displayed in color and white contours. The radio continuum emission as observed at $1.4 \mathrm{GHz}$ is shown in green contours. Note the open HI shell surrounding the northern and western sides of the SNR and opening towards the south-east, where the SNR vanishes. The high-energy source HESS J1702-420 lies outside this figure, towards the lower right corner.

column density was calculated from $N_{\mathrm{H}}=2 N\left(\mathrm{H}_{2}\right)+N(\mathrm{HI})$. To estimate the $\mathrm{H}_{2}$ column density, we extracted the ${ }^{12} \mathrm{CO}$ data from the CfA CO survey (Dame et al. 2001). In this estimation we assume local thermodynamic equilibrium and we adopt a molecular mass calibration ratio $X=2.7 \times 10^{20} \mathrm{~cm}^{-2}$ (Dame et al. 2001). The hydrogen column density obtained to a distance of $6.3 \mathrm{kpc}$ is $\sim 4 \times 10^{22} \mathrm{~cm}^{-2}$, where we take into account the distance ambiguity by assuming that $2 / 3$ of the total gas emission comes from the near side and $1 / 3$ from the far side of our Galaxy. This value is consistent with the absorbing column density estimated from our spectral fit of XMM-Newton data for the whole remnant $\left(4.5 \pm 0.2 \times 10^{22} \mathrm{~cm}^{-2}\right.$, see Sect. 3.2.2).

\subsubsection{The $\mathrm{HI}$ shell around $\mathrm{G} 344.7-0.1$}

In what follows we investigate the origin of the neutral shell based on its characteristic parameters. It is centred at $\sim 17^{\mathrm{h}} 03^{\mathrm{m}} 55^{\mathrm{s}} .6,-41^{\circ} 42^{\prime} 28^{\prime \prime} .6(\sim 344.7,-0.17$ in Galactic coordinates) and its radius is $\sim 8^{\prime}(\sim 14.6 \mathrm{pc}$ at the distance of $6.3 \mathrm{kpc})$. By assuming optically thin gas and integrating the column density between -119 and $-110 \mathrm{~km} \mathrm{~s}^{-1}$, the total mass in the shell is estimated in about $1300 M_{\odot}$ and the kinetic energy injected into the ISM is about $3 \times 10^{47} \mathrm{erg}$. This last value was calculated by assuming an expansion velocity of $\sim 7 \mathrm{~km} \mathrm{~s}^{-1}$, as suggested from the HI data. This expansion velocity is a lower limit, since confusion from unrelated foreground and background emission impedes the detection of possible caps of the expanding shell. Assuming a linear expansion regime as a first approximation, we estimate the age of the HI feature to be about $10^{6} \mathrm{yr}$, orders of magnitude older than the $\sim 3000 \mathrm{yr}$ estimated for this SNR on the basis of our X-ray study. Therefore we are led to the conclusion that the detected cold neutral envelope is the neutral gas swept up by the stellar wind of the precursor star.

Wind blown HI-shells such as the one detected around G344.7-0.1 are common and result from the effect of massive stars on the ISM (e.g., Giacani \& Dubner 2004; Cappa et al. 2005; Cichowolski et al. 2008). To test this hypothesis we first compare the kinetic energy of the HI shell structure with the mechanical energy released by a star into the ISM during the lifetime of the bubble, as calculated with the equation 

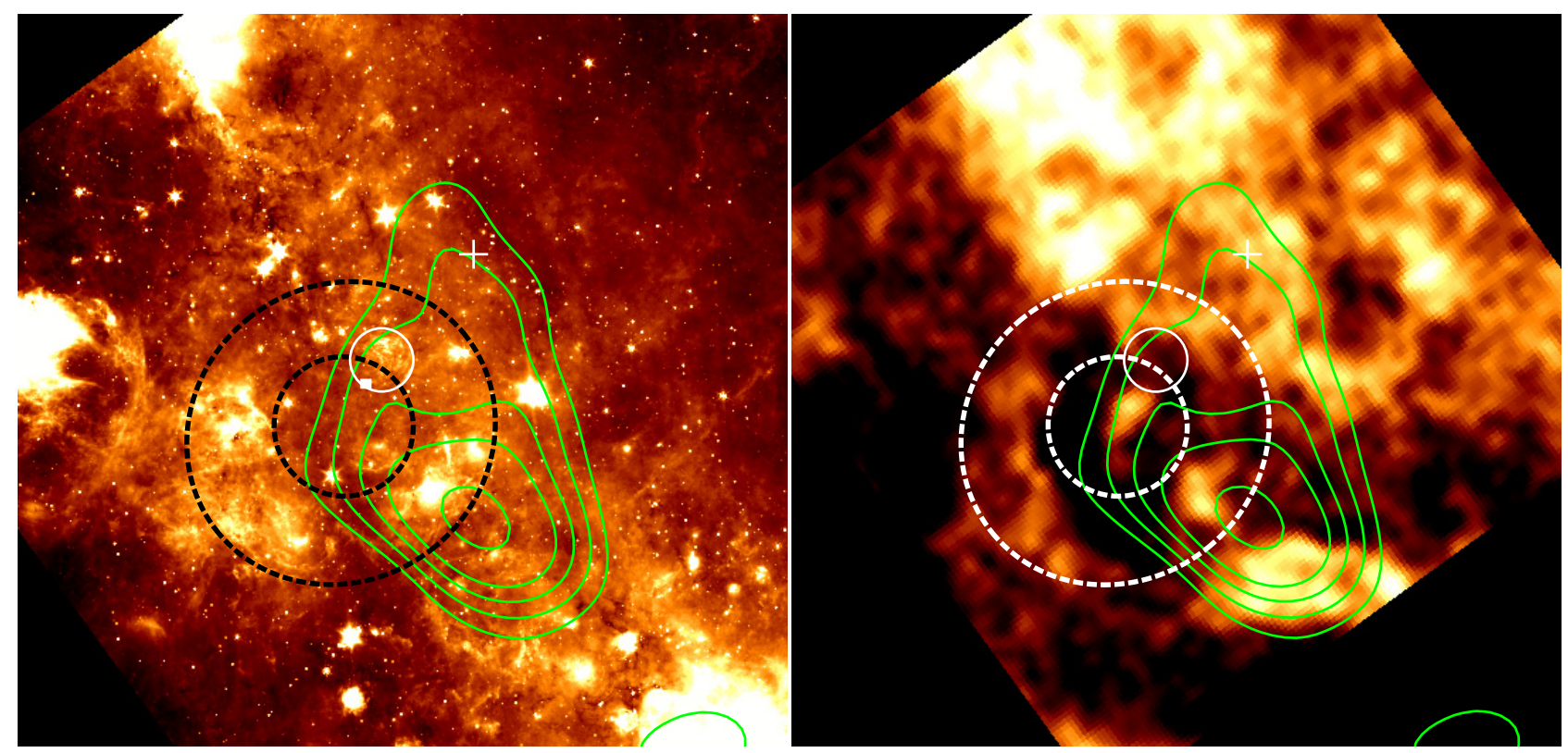

Fig. 6. Left: large-scale distribution of the IR $24 \mu \mathrm{m}$ emission; Right: HI distribution traced at $v_{\mathrm{LSR}} \sim 115 \mathrm{~km} \mathrm{~s}^{-1}$. Both images are presented in equatorial coordinates. HESS J1702-420 significance contours are shown in green, the small white circle shows the location and size of G344.7-0.1, the white plus sign marks the position of PSR J1702-4128. The dotted circles are discussed in the text.

$E_{w}=10^{43} \dot{M} V_{\infty}^{2} t_{\mathrm{dyn}} / 2$, where $\dot{M}$ is the mass-loss rate of the star in $M_{\odot} / \mathrm{yr}, V_{\infty}$ is the terminal velocity of the wind in $\mathrm{km} \mathrm{s}^{-1}$, and $t_{\text {dyn }}$ is the dynamical age of the structure in years. If we assume that only one star has powered the observed bubble, a single O9 V star with a mean mass-loss rate of $\dot{M} \sim 1.5 \times 10^{-7} M_{\odot} / \mathrm{yr}$ (de Jager et al. 1988; Mokiem et al. 2007) and a wind velocity of about $2000 \mathrm{~km} \mathrm{~s}^{-1}$ (Prinja et al. 1990) would be sufficient to sweep up the amount of interstellar gas observed to have accumulated in the shell, after taking into account that the energy conversion efficiency in a bubble is no more than $20 \%$ (see Koo $\&$ McKee 1992). In this case, the mechanical energy released by this star into the ISM is about $6 \times 10^{47} \mathrm{erg}$, enough to explain the calculated kinetic energy of the slowly expanding HI shell $\left(3 \times 10^{47} \mathrm{erg}\right)$. These results are independently confirmed by applying equation 2 in the model discussed by Chevalier (1999).

In this scenario the SNR G344.7-0.1 would be the result of a core-collapse explosion (type Ib or type II) expanding inside the wind-blown bubble created by the pre-supernova star. This fact, together with the relatively young age derived, can explain the spherical symmetry that still holds this SNR.

\subsection{The big picture: the SNR G344.7-0.1 and HESS J1702-420}

To investigate the likelihood of a connection between the H.E.S.S. source and the SNR G344.7-0.1, we examined the properties of the ambient interstellar medium. To this end we analysed the distribution of the $\lambda 24 \mu \mathrm{m}$ infrared emision, which serves to trace the sites of shocked dust, and of the HI at the systemic velocity of G344.7-0.1 within a large field around HESS J1702-420. In Fig. 6 we show the H.E.S.S. significance contours (in green) overlapping the emission distribution as observed in both spectral regimes. The small white circle indicates the location and size of the SNR G344.7-0.1. A quick inspection reveals that the SNR lies on a large circular ring of enhanced emission visible in both spectral regimes. The dotted circles are included to aid the visualization of the feature mentioned. Particularly in IR, the ring-like feature appears defined by a chain of bright spots. The small, open HI shell shown in Fig. 5, proposed to be associated with G344.7-0.1, can now be appreciated as being part of a larger ring-like structure, a thick shell that also intersects the maximum of HESS J1702-420.

The characteristic parameters of this big shell can be summarized as follows: center $\sim 17^{\mathrm{h}} 04^{\mathrm{m}} 21^{\mathrm{s}} .8,-41^{\circ} 52^{\prime} 30^{\prime \prime}(\sim 344.6$, -0.34 in Galactic coordinates); outer radius $\sim 21^{\prime}(\sim 38.5 \mathrm{pc}$ at the distance of $6.3 \mathrm{kpc})$, inner radius $\sim 9^{\prime}(\sim 16.5 \mathrm{pc})$; associated HI mass $\sim 7400 M_{\odot}$; expansion velocity $\sim 5 \mathrm{~km} \mathrm{~s}^{-1}$; kinetic energy $E=1.8 \times 10^{48} \mathrm{erg}$; age $=2.6 \times 10^{6} \mathrm{yr}$.

We also inspected the distribution of the ${ }^{12} \mathrm{CO}$ gas, especially looking for high-density clouds near the SNR where the nucleonic cosmic ray components can interact via $\pi^{0}-$ mesons to produce the observed gamma-ray emission. We used the only publicly available ${ }^{12} \mathrm{CO}$ data in this region, the Galactic CO survey by Dame et al. (2001), which maps the molecular gas emission with $8^{\prime}$ resolution $(\sim 15 \mathrm{pc}$ at the distance of $6.3 \mathrm{kpc})$, and investigated the ${ }^{12} \mathrm{CO}$ distribution near the LSR velocities $\sim-110$ to $\sim-130 \mathrm{~km} \mathrm{~s}^{-1}$.

Figure 7 shows the ${ }^{12} \mathrm{CO}$ distribution in a large area around the gamma ray source at the kinematical velocity of the SNR. It can be seen that HESS J1702-420 lies over low temperature $(\simeq 0.4 \mathrm{~K})$ molecular matter, in between two brighter concentrations. Such cloud, in principle, does not seem to be a good target to produce gamma ray emission through hadronic mechanism. However, since the angular resolution of the molecular data is too poor to identify small, very dense clumps that can be immersed in a more tenuous medium (see for example, Paron \& Giacani 2010), we can not discard this possibility. In fact, the presence of small size molecular concentrations overrun by the SN shock was demostrated in Sect. 3.2.1. Clumps of comparable size of the one suggested to be shocked by G344.7-0.1 producing the central nebula, are completely overlooked with a beam size over 3 times bigger. We note that around the LSR velocity of $-25 \mathrm{~km} \mathrm{~s}^{-1}$ (distance of 2.4 or $13 \mathrm{kpc}$ ) there is an extended, slightly brighter ${ }^{12} \mathrm{CO}$ structure that overlaps HESS J1702-420 and beyond. If this molecular matter were responsible for the gamma-ray emission observed in HESS J1702-420, 


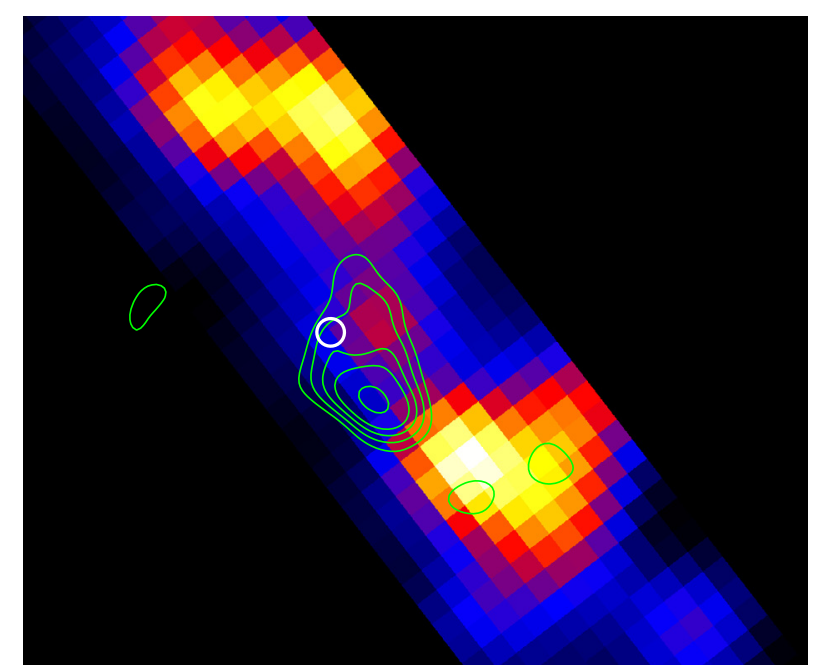

Fig. 7. The ${ }^{12} \mathrm{CO}$ distribution in a large area around HESS J1702-420 (green contours) at the systemic velocity of G344.7-0.1. The white circle again represents location and size of the SNR.

then another still unidentified cosmic ray accelerator should be found at a compatible distance.

Nevertheless, in spite of the no detection of a dense molecular cloud associated with G344.7-0.1 and HESS J1702-420, a reasonable scenario that takes into account the various observational facts would be the following: a massive star (or stars) of a former generation swept up the large ring of gas via stellar wind. A second generation of stars was born in this large shell and, about $3000 \mathrm{yr}$ ago, one of them exploded creating the SNR G344.7-0.1. This SNR is, in turn, interacting with the wind bubble blown up by its own precursor and/or the inhomogeneous gas that forms the larger ring. This scenario basically describes an active region, where the interstellar gas has been disturbed and stirred by stellar winds, stellar births and stellar deaths for over a couple of million of years, suggesting that G344.7-0.1 and its peculiar environment can be the origin of the observed gamma rays.

\section{Conclusions}

In this paper we have revisited the SNR G344.7-0.1 producing new, high-quality images in radio and a new analysis of X-ray data. We have also investigated the likelihood of a physical connection between this SNR, as a cosmic ray accelerator, and the high-energy source HESS J1702-420 located in its vicinity.

The new radio images of G344.7-0.1 have revealed that the central nebula is very structured, with small-scale features. From a spectral study carried out in this region of the remnant, we determined a mean radio spectral index for the synchrotron radiation of $\alpha \sim-0.3$, flatter than the global spectral index of the remnant. Though this value is compatible with those of radio PWNe, this hypothesis is weakened by the lack of any other evidence supporting this origin, while a straightforward interpretation arise from the multiwavelength images of G344.7-0.1. In fact, from the combination of radio, mid-IR and X-ray observations, we conclude that this central bright feature is the result of strong shocks impacting on a dense wall, which is manifested as an enhancement of the radio synchrotron emission and IR emission (from shocked dust) and produces a flattening in the radio spectrum.

The XMM-Newton image of this SNR indicates that the $\mathrm{X}$-ray emission is characterized by a centrally filled X-ray morphology and faint diffuse emission that extends up the radio boundary. The X-ray emission distribution is complex, formed by arcs and filaments. Our study confirms its thermal nature, with no evidence for non-thermal synchrotron emission and with enhanced metal abundances indicative of ejecta origin.

Taking into account both the observed morphology in radio and X-rays and the thermal nature of the X-ray emission, we propose that G344.7-0.1 belongs to the class of mixed-morphology (MM) remnants (Rho \& Petre 1998) and, within this group, to the emerging sub-class of metal-rich MM remnants. Although from the available low-resolution ${ }^{12} \mathrm{CO}$ data we did not find an associated dense molecular cloud, the $\mathrm{Mg} / \mathrm{Si}$ and $\mathrm{Ar} / \mathrm{Si}$ ratios derived from our X-ray spectral fit, favour a core-collapse origin for G344.7-0.1. It is known that this type of SNe originates from massive stars usually located not far from the dense molecular complexes of their birth. Besides, all the MM SNRs reported up to now have been found to be interacting with molecular clouds.

The distance to the SNR G344.7-0.1 was recalculated using different methods, resulting in a value of $6.3 \pm 0.1 \mathrm{kpc}$. The new distance is perfectly compatible with the distance estimate based on the X-ray absorbing column. An age of about $3000 \mathrm{yr}$ is derived for G344.7-0.1.

No radio counterpart has been discovered for CXOU J170357.8-414302, the X-ray point source reported by Combi et al. (2010).

Concerning the existence of a possible connection between G344.7-0.1 and the "dark" gamma-ray source HESS J1702420 , from the study of the distribution and kinematics of the atomic gas in the surroundings of both sources, we propose that the SNR G344.7-0.1 is expanding within a wind-blown bubble created by its massive progenitor. The pre-supernova star has swept up an HI shell that is partially surrounding the SNR along its brighter limbs and opens in the direction where the SNR appears fainter. This neutral shell is, in turn, part of a larger expanding ring visible in HI and in IR $24 \mu \mathrm{m}$, with an age of a couple of million years. We propose that the stellar wind of a massive star (or stars) created this large structure. A second generation of stars formed in this compressed gas, and about $3000 \mathrm{yr}$ ago one of these stars exploded, giving origin to the now observable SNR G344.7-0.1. This scenario describes a turbulent region, where the gas has been stirred by stellar winds, star formation and explosions along millions of years. The presence of a cosmic ray accelerator, such as the SNR shock, acting on turbulent molecular matter as target for accelerated hadrons, can provide the long searched explanation for the gamma-rays observed here. Future works are planned to complete the molecular sudies, searching for small, dense clumps inmmersed in the tenuous molecular gas detected, providing the nuclei responsible for the production of neutral pions, which decay yielding the observed gamma-rays. Also dedicated observations in the GeV range performed with the Fermi/LAT instrument will be very useful to confirm the nature of the gamma-ray emission.

Acknowledgements. E. Giacani and G. Dubner are members of the Carrera del Investigador Cientifico of CONICET, Argentina. We thanks the anonymous referee for helful comments. E.G. acknowledges support from the Faculty of the European Space Astronomy Centre (ESAC). This research was partially funded by Argentina Grants awarded by ANPCYT, CONICET and University of Buenos Aires (UBACYT A023). This work is based on observations done with the $X M M-N e w t o n$, an ESA science mission with instruments and contributions directly funded by ESA Member States and the US (NASA). 


\section{References}

Aharonian, F., Akhperjanian, A. G., Bazer-Bachi, A. R., et al. 2006a, A\&A, 449, 223

Aharonian, F., Akhperjanian, A. G., Bazer-Bachi, A. R., et al. 2006b, ApJ, 636, 777

Aharonian, F., Akhperjanian, A. G., Barres de Almeida, U., et al. 2008, A\&A, 477,353

Anders, E., \& Grevesse, N. 1989, Geochim. Cosmochim. Acta, 53, 197

Borkowski, K. J., Lyerly, W. J., \& Reynolds, S. P. 2001, ApJ, 548, 820

Cappa, C., Niemela, V. S., Martín, M. C., \& McClure-Griffiths, N. M. 2005, A\&A, 436, 155

Castro, D., Slane, P., Patnaude, D. J., \& Ellison, D. C. 2011, ArXiv e-prints

Chang, C., Konopelko, A., \& Cui, W. 2008, ApJ, 682, 1177

Chevalier, R. A. 1974, ApJ, 188, 501

Chevalier, R. A. 1999, ApJ, 511, 798

Cichowolski, S., Pineault, S., Arnal, E. M., \& Cappa, C. E. 2008, A\&A, 478, 443

Combi, J. A., Albacete Colombo, J. F., López-Santiago, J., et al. 2010, A\&A, 522, A50

Costain, C. H. 1960, MNRAS, 120, 248

Dame, T. M., Hartmann, D., \& Thaddeus, P. 2001, ApJ, 547, 792

de Jager, C., Nieuwenhuijzen, H., \& van der Hucht, K. A. 1988, A\&AS, 72, 259

Dubner, G. M., Moffett, D. A., Goss, W. M., \& Winkler, P. F. 1993, AJ, 105, 2251

Ellison, D. C., Slane, P., \& Gaensler, B. M. 2001, ApJ, 563, 191

Enomoto, R., Tanimori, T., Naito, T., et al. 2002, Nature, 416, 823
Gaensler, B. M., \& Slane, P. O. 2006, ARA\&A, 44, 17

Giacani, E., \& Dubner, G. 2004, A\&A, 413, 225

Giacani, E., Loiseau, N., Smith, M. J. S., Dubner, G., \& Iacobelli, M. 2010, AIP

Conf. Ser. 1248, ed. A. Comastri, L. Angelini, \& M. Cappi, 39

Green, D. A. 2009, VizieR Online Data Catalog, 7253

Harrus, I. M., Slane, P. O., Smith, R. K., \& Hughes, J. P. 2001, ApJ, 552, 614

Koo, B.-C., \& McKee, C. F. 1992, ApJ, 388, 93

Mazzotta, P., Mazzitelli, G., Colafrancesco, S., \& Vittorio, N. 1998, A\&AS, 133, 403

McClure-Griffiths, N. M., Dickey, J. M., Gaensler, B. M., et al. 2005, ApJS, 158, 178

Mokiem, M. R., de Koter, A., Vink, J. S., et al. 2007, A\&A, 473, 603

Muraishi, H., Tanimori, T., Yanagita, S., et al. 2000, A\&A, 354, L57

Park, S., Slane, P. O., Hughes, J. P., et al. 2007, ApJ, 665, 1173

Paron, S., \& Giacani, E. 2010, A\&A, 509, L4

Prinja, R. K., Barlow, M. J., \& Howarth, I. D. 1990, ApJ, 361, 607

Rho, J., \& Petre, R. 1998, ApJ, 503, L167

Rieke, G. H., Young, E. T., Engelbracht, C. W., et al. 2004, ApJS, 154, 25

Smith, R. K., Brickhouse, N. S., Liedahl, D. A., \& Raymond, J. C. 2001, ApJ, 556, L91

Taylor, J. H., Manchester, R. N., \& Lyne, A. G. 1993, ApJS, 88, 529

Turtle, A. J., Pugh, J. F., Kenderdine, S., \& Pauliny-Toth, I. I. K. 1962, MNRAS, 124,297

Whiteoak, J. B. Z., \& Green, A. J. 1996, A\&AS, 118, 329

Yamauchi, S., Ueno, M., Koyama, K., \& Bamba, A. 2005, PASJ, 57, 459 